\title{
2018 Reviewer Acknowledgement
}

\section{Editorial Office of Exploratory Research and Hypothesis in Medicine}

We thank the following reviewers for their contribution and support in 2018 .

\author{
Sarah Farukhi Ahmed \\ United States \\ Ali Akdogan \\ Turkey \\ Sudheer Arava \\ India \\ Miguel Borja \\ Mexico \\ Christopher Brooks \\ United States \\ Chang Chen \\ United States \\ Bohao Chen \\ United States \\ Patricia M Crittenden \\ United States \\ Monica De la Fuente \\ Spain \\ Ahmed M Elsodany \\ Egypt \\ Engin Erturk \\ Turkey \\ Ezzatollah Fathi
}

Iran

Yair Feld

Israel

Alfredo Franco-Obregón

Singapore

Jamil Ghahhari

Italy

Nianqiao Gong

China

Sanna Isosävi

Finland

Hans O Kalkman

Switzerland

Abbas Khani

Switzerland

Tae Ho Lee

United States

Fernando Lopes

Canada

Susumu Matsukuma

Japan

David Miller

United Kingdom
Ahmed E. Abdel Moneim

Egypt

Sabina Passamonti

Italy

Sedat Saylan

Turkey

You Shang

China

Anand Singh

United States

Tomoyasu Sugiyama

Japan

Hemant D. Une

Australia

Yu N Utkin

Russia

Lanjing Zhang

United States

Weilin Zhang

China

Peng Zhang

China

DOI: 10.14218/ERHM.2018.000RA 